\title{
Scanning gate microscopy of localized states in a gate-defined bilayer graphene channel
}

\author{
Carolin Gold $\odot,{ }^{1, *}$ Annika Kurzmann, ${ }^{1}$ Kenji Watanabe $\odot,{ }^{2}$ Takashi Taniguchi, ${ }^{3}$ Klaus Ensslin $\odot,{ }^{1}$ and Thomas Ihn ${ }^{1}$ \\ ${ }^{1}$ Solid State Physics Laboratory, ETH Zürich, CH-8093 Zürich, Switzerland \\ ${ }^{2}$ Research Center for Functional Materials, National Institute for Materials Science, 1-1 Namiki, Tsukuba 305-0044, Japan \\ ${ }^{3}$ International Center for Materials Nanoarchitectonics, National Institute for Materials Science, 1-1 Namiki, Tsukuba 305-0044, Japan
}

(Received 29 July 2020; revised 9 November 2020; accepted 16 November 2020; published 16 December 2020)

\begin{abstract}
We use scanning gate microscopy to demonstrate the presence of localized states arising from potential inhomogeneities in a $50 \mathrm{~nm}$ wide, gate-defined conducting channel in encapsulated bilayer graphene. When imaging the channel conductance under the influence of a local tip-induced potential, we observe ellipses of enhanced conductance as a function of the tip position. These ellipses allow us to infer the location of the localized states and to study their dependence on the displacement field. For large displacement fields, we observe that localized states tend to occur halfway into the channel. All our observations can be well explained within the framework of stochastic Coulomb blockade.
\end{abstract}

DOI: 10.1103/PhysRevResearch.2.043380

\section{INTRODUCTION}

Graphene is a promising material for semiconducting quantum-dot-based qubits due to its small spin-orbit coupling and small hyperfine interaction [1]. For a long time, the fabrication of high-quality graphene-based nanodevices was hindered, e.g., by the presence of strong background potential modulations arising from the inhomogeneities and surface roughness of the silicon oxide substrates, as well as the existence of localized states at the etched device boundaries [2-5]. Only recently, the conjunction of two key technological advancements has enabled the reproducible fabrication of high-quality graphene nanodevices. First, using encapsulation between hexagonal boron nitride (hBN) flakes [6], edge contacting [7], and graphite back gates [8] enabled the fabrication of single- and bilayer graphene devices with very high electronic quality. Second, the use of bilayer graphene with its electrostatically tunable band gap provided the possibility to create gate-defined devices [9-11]. These devices require an average disorder potential smaller than the gate-induced band gap. If the latter condition is met, their smooth edge potentials in comparison to the previously etched devices minimize the appearance of localized edge states. This made it possible to prepare high-quality quantum point contacts $[8,12]$ as well as few-electron or few-hole quantum dots in bilayer-graphene channels $[13,14]$. These devices allow observing a plethora of intriguing phenomena such as a tunable valley splitting in quantum point contacts [15], excited states in few-electron or few-hole quantum dots [16], and charge detection [17].

\footnotetext{
*cgold@phys.ethz.ch

Published by the American Physical Society under the terms of the Creative Commons Attribution 4.0 International license. Further distribution of this work must maintain attribution to the author(s) and the published article's title, journal citation, and DOI.
}

However, the variability in the conductance properties of nominally identical devices fabricated in close proximity to each other indicates that local inhomogeneity of the electrostatic potential and disorder may still play an important role. To date, no experiments exist that focus on the local and systematic study of inhomogeneities and the possible formation of localized states in such devices.

A powerful technique to engineer and image localized states in samples in which the graphene is accessible at the surface is scanning tunneling microscopy [18,19]. Due to the hBN-encapsulation and the presence of gates, however, this technique is not viable on gate-defined bilayer graphene devices. In this paper we use scanning gate microscopy (SGM) to demonstrate the formation of localized states in a gate-defined bilayer graphene channel. Scanning the voltagebiased metallic tip of an atomic force microscope over the channel perturbs the potential landscape locally. This technique has previously been used to image localized states, both disorder induced as well as engineered, in systems as diverse as InAs nanowires [20], GaAs heterostructures [21,22], carbon nanotubes [23-26], and graphene [27-29]. References $[28,29]$ used the scanning gate technique on single-layer graphene directly deposited on a silicon oxide substrate and dry etched into a narrow constriction [28] or a quantum dot [29]. In contrast, our experiment is based on gate-defined bilayer graphene devices that have been shown to significantly reduce both substrate $[6,8]$ as well as edge disorder and localization $[8,9]$. We investigate a channel which is narrower $(50 \mathrm{~nm})$ than the typical channel widths used for quantum dot studies $(100 \mathrm{~nm})$. When the conductance through the channel is almost completely suppressed in the absence of the tip, we observe conductance resonances associated with localized states. These resonances lead to concentric rings or ellipses in the two-dimensional conductance maps obtained in SGM measurements. The center points of these ellipses allow us to infer the position of the localized states. By varying the displacement field forming the channel, we furthermore gain 

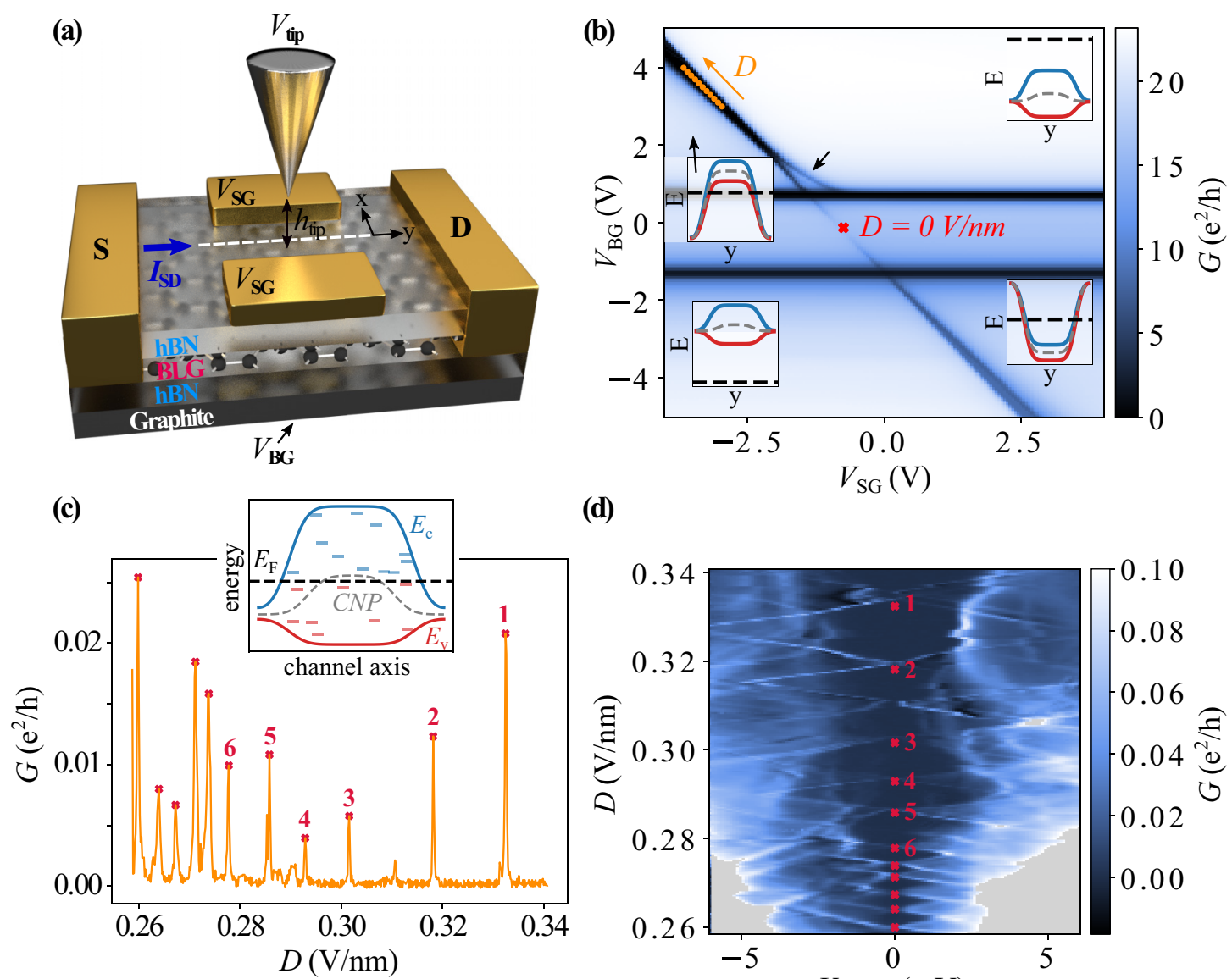

(d)



FIG. 1. (a) Schematic of the measurement setup consisting of sample plus SGM tip. The sample consists of an encapsulated bilayer graphene (BLG) on a graphite back gate. Displacement fields between split gates and graphite back gate form a channel. The voltage-biased tip is scanned $55 \mathrm{~nm}$ (with respect to the top hBN-layer surface) above the channel while we measure the conductance $G$ between source (S) and drain (D). (b) Two-terminal linear conductance $G$ as a function of split-gate voltage $V_{\mathrm{SG}}$ and back-gate voltage $V_{\mathrm{BG}}$ in the absence of the tip. The diagonal line corresponds to charge neutrality underneath the split gates. The displacement field in the double-gated areas increases along the dark-orange arrow with respect to $D=0 \mathrm{~V} / \mathrm{nm}$ denoted by the red cross. The insets depict the energy of the conduction band (blue), valence band (red), charge neutrality point (grey), and Fermi-Energy (dashed black line) along the white dashed line in (a) for the respective gate configurations. (c) Conductance as a function of the displacement field $D$ along the charge neutrality point [orange dotted line in (b)]. The inset depicts the schematic of a possible potential landscape along the channel axis [dashed white line in $(\mathrm{a})]$. $E_{\mathrm{c}}\left(E_{\mathrm{v}}\right)$ denotes the conduction (valence) band, $E_{\mathrm{F}}$ the Fermi energy and CNP the charge neutrality point. (d) Coulomb diamonds. The grey regions correspond to conductance values exceeding the upper limit of the color scale.

insights into the evolution of the localized states as a function of the band-gap size and electron concentration.

\section{SAMPLE CHARACTERIZATION}

We perform measurements on the van-der Waals stack depicted schematically in Fig. 1(a). It consists of a bilayer graphene (BLG) flake encapsulated between two hBN flakes of different thicknesses $t \quad\left(t_{\text {top-hBN }}=32 \mathrm{~nm}, t_{\text {bottom-hBN }}=\right.$ $40 \mathrm{~nm}$ ) and deposited on top of a graphite back gate (BG). The fabrication of the device follows the process outlined in Ref. [8]. Ohmic source (S) and drain (D) contacts connect to the BLG sheet while lithographically defined split gates (SG, $300 \mathrm{~nm}$ long and $50 \mathrm{~nm}$ apart) allow us to confine the current flow in the graphene to a channel between the split gates. Throughout this work, the same voltage $V_{\mathrm{SG}}$ is applied to both split gates, and the back gate bias is given by the voltage $V_{\mathrm{BG}}$. A displacement field $D=\left[C_{\mathrm{BG}}\left(V_{\mathrm{BG}}-V_{\mathrm{BG}}^{0}\right)-C_{\mathrm{SG}}\left(V_{\mathrm{SG}}-V_{\mathrm{SG}}^{0}\right)\right] / 2 \epsilon_{0}$ is generated in the double-gated areas by applying a voltage difference between split gate (SG) and back gate (BG). Here, $C_{\mathrm{SG}}$ and $C_{\mathrm{BG}}$ are the respective gate-graphene capacitances per unit area of the split gate (SG) and back gate (BG) [30]. The charge neutrality point in the double-gated area [red cross in Fig. 1(b)] occurs at the offset voltages $V_{\mathrm{SG}}^{0}=-0.744 \mathrm{~V}$ and $V_{\mathrm{BG}}^{0}=-0.13 \mathrm{~V}$. As the gate voltages are tuned away from these voltages, the thus generated displacement field opens a band gap in the bilayer graphene below the split-gated regions and in the channel. The average of split- and back-gate voltages weighted by the gate capacitances furthermore allows us to tune the Fermi level in the graphene sheet below the split gates into the band gap, rendering it insulating. This results in a narrow channel consisting of the remaining conductive region between the gates. 
All our measurements are performed at $T=270 \mathrm{mK}$ in a ${ }^{3} \mathrm{He}$ cryostat. By applying both an $\mathrm{AC}$ voltage $V_{\mathrm{SD}, \mathrm{AC}}=$ $50 \mu \mathrm{V}_{\text {rms }}$ as well as a variable DC voltage $V_{\mathrm{SD}, \mathrm{DC}}$ between the source (S) and drain (D) contacts, we can perform both linear and nonlinear transport measurements. To this end, we measure the differential conductance $G=I_{\mathrm{SD}, \mathrm{AC}} / V_{\mathrm{SD}, \mathrm{AC}}$ using a home-built $I V$ converter and a low-frequency lock-in. The thus obtained conductance $G$ corresponds to the sample conductance $G_{\mathrm{S}}$ for the highly resistive sample configuration used throughout all our experiments, except for the highly conductive regions in Fig. 1(b). In the latter, $G$ constitutes the lower limit of the sample conductance, due to the presence of cable-, filter-, and contact resistances connected in series to the sample. To explore the local properties of the channel, we perform SGM measurements. As depicted in Fig. 1(a), we thus position a voltage-biased metallic tip at a height $h_{\text {tip }}=55 \mathrm{~nm}$ above the top $\mathrm{hBN}$ surface in the area above the channel. By raster-scanning the tip we measure the differential conductance $G$ as a function of the tip position $(x, y)$ or the tip voltage $V_{\text {tip }}$.

To demonstrate the formation of the channel experimentally, we first characterize the combined effect of the back-gate voltage $V_{\mathrm{BG}}$ and split-gate voltage $V_{\mathrm{SG}}$ on the conductance through the device. Figure 1(b) depicts the linear conductance $G\left(V_{\mathrm{SG}}, V_{\mathrm{BG}}\right)$. The latter depends on the configuration of the band structure in the sample along the white-dashed line in Fig. 1(a). The varying effect of the gate-voltages tunes the conductance and valence band as well as the charge neutrality point in the the single-gated $\left(V_{\mathrm{BG}}\right)$ regions outside the channel and the dual-gated channel region $\left(V_{\mathrm{SG}}, V_{\mathrm{BG}}\right)$ as depicted in the insets of Fig. 1(b). The diagonal conductance minimum depending on both $V_{\mathrm{BG}}$ and $V_{\mathrm{SG}}$ corresponds to charge neutrality of the area underneath the split gates. Its depth depends nonmonotonically on the displacement field $D$, reflecting the electrostatic landscape in the channel. The zero of the displacement field is the point of maximum conductance along this line, which is marked by the red cross in Fig. 1(b). For all gate-voltages along the conductance minimum, the split-gated areas have zero total density and tend to be increasingly insulating for increasing displacement fields due to the increasing band gap in the dual-gated regions. Therefore the source-drain current is confined to the channel at sufficiently large displacement fields along this minimum.

In addition, we observe two horizontal, split-gate independent conductance minima. The small additional feature marked by the black arrow in Fig. 1(b) indicates that the horizontal conductance minimum at positive $V_{\mathrm{BG}}$ most likely arises due to the charge neutrality point of the bulk regions around the channel. The minimum at negative $V_{\mathrm{BG}}$ belongs to the charge neutrality point of additional devices connected in series to that of interest here, which are not used in the present work.

In contrast to previous measurements on samples with mostly larger channel widths [8,31], the formation of the channel not only reduces the conductance but suppresses it almost completely for the displacement fields marked by the orange dotted line in Fig. 1(b). Along this line, the bulk regions of the charge carrier gas outside the channel are $n$-type with a density larger than $1 \times 10^{12} \mathrm{~cm}^{-2}$, while the double-gated areas are at zero density with the Fermi level in the middle of the band gap. In contrast to the double-gated regions, the channel region only couples to the split gates via stray fields. As the electrostatic coupling of the channel is thus dominated by the back gate, the electron density in the channel increases with increasing displacement field along the orange line of reduced conductance [ $D$ arrow in Fig. 1(b)]. The experimental fact that the device reaches zero conductance at positive displacement field values suggests that charge neutrality in the channel occurs at these positive displacement fields. This is further corroborated by the asymmetric suppression of the conductance along the diagonal line with respect to positive and negative displacement fields $D$.

Measuring the conductance along the orange dotted line in Fig. 1(b) with high resolution reveals the conductance resonances of different spacing and height shown in Fig. 1(c). To identify the same peaks throughout the measurements presented in this paper, we label them from 1 to 6 . The observation of such strong conductance resonances suggests that localized states exist inside the channel region, which transmit resonantly once their energy coincides with the Fermi level.

This interpretation is confirmed by the finite-bias spectroscopy measurements in Fig. 1(d), which shows diamonds of suppressed conductance and irregular size. They represent the fingerprints of one or more localized states in the channel. The presence of low-energy excited state resonances outside the diamonds of suppressed conductance supports the interpretation in terms of Coulomb blockade. The diamond between resonances 1 and 2 has an extent of $6.3 \mathrm{mV}$ in the $V_{\mathrm{SD}, \mathrm{DC}}$ direction. Assuming that the entire voltage drops across this single quantum-dot-like impurity leads to an energy calibration factor for the displacement field axis of $420 \mathrm{meV} /(\mathrm{V} / \mathrm{nm})$. Figure 1(d) therefore spans an energy range of about $34 \mathrm{meV}$, which is the same order of magnitude as typical energy gaps expected at these displacement fields [32-34].

The charging energy for increasing electron numbers grows from $2 \mathrm{meV}$ to more than $6 \mathrm{meV}$ with increasing displacement field. This finding suggests that the channel tends to become more insulating with increasing displacement field, presumably due to the increasing energy gap and the Fermi energy moving closer to the center of the gap.

The schematic in the inset of Fig. 1(c) summarizes one possible understanding of the device along the white dashed line in Fig. 1(a). Here, $E_{\mathrm{v}}\left(E_{\mathrm{c}}\right)$ denotes the energy of the valence (conduction) band edge along the channel axis, $E_{\mathrm{F}}$ is the Fermi energy and CNP the charge neutrality point. Inhomogeneities of the potential induce localized states in the gap as indicated schematically by the horizontal lines within the band gap. These inhomogeneities can arise due to a variety of sources, including, e.g., the exact shape of the channel gates as well as defects in the graphene sheet, the $\mathrm{hBN}$, or between the different layers of the van-der-Waals heterostructure. Depending on their position with respect to the charge neutrality point, these localized states can have stronger valence band character [light-red lines in the inset of Fig. 1(c)] or stronger conduction band character [light-blue lines in the inset of Fig. 1(c)]. Electrons occupying these states may tunnel from the channel into the high-density regions outside the channel. Localized states halfway along the channel axis will typically have the highest transmission 


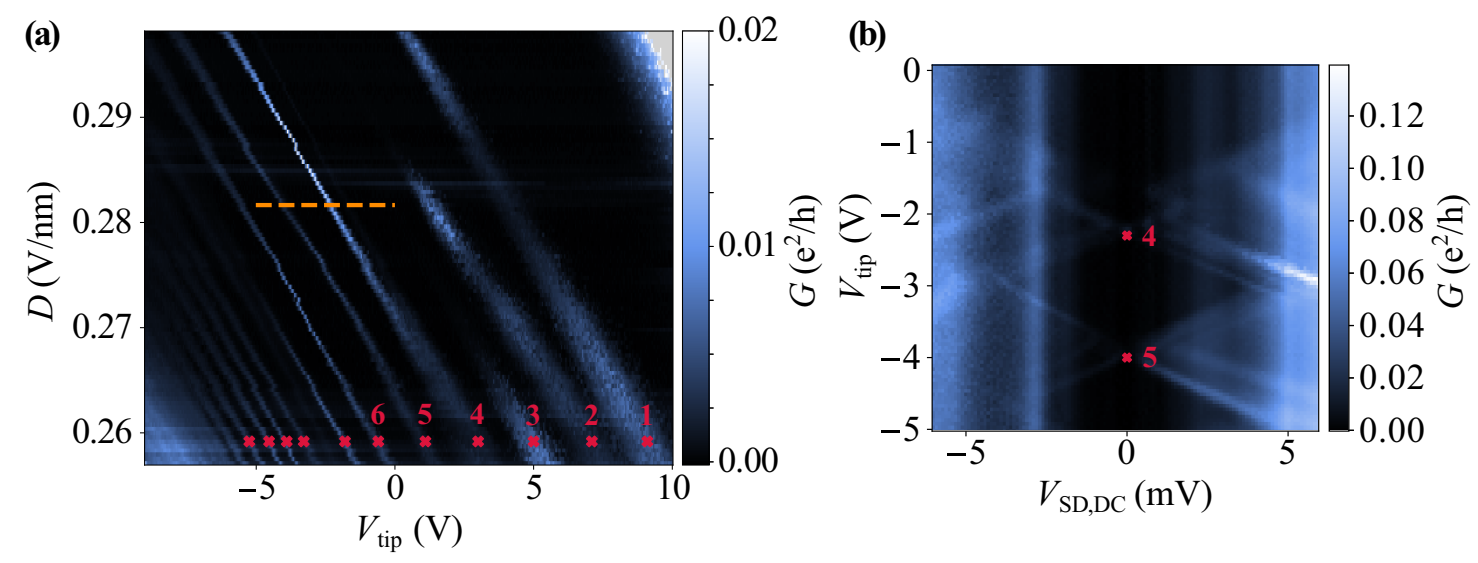

FIG. 2. Measurement at fixed lateral tip position above the channel. (a) Two-terminal conductance as a function of tip voltage $V_{\text {tip }}$ and displacement field $D$ along the charge neutrality point [orange line in Fig. 1(b)]. Conductance values exceeding the upper limit of the color scale are depicted in grey. (b) Coulomb diamonds measured as a function of tip voltage $V_{\text {tip }}$ at the displacement field denoted by the orange dashed line in (a).

in the case where a single state dominates the conductance of the whole channel. An alternative schematic would show the effect of disorder and the appearance of localized states in terms of spatially varying band-edge energies. In such a scenario, localized states would form in potential pockets of either the conduction or the valence band edge.

In such a model, localized states would be distributed in an uncontrolled way both in position and energy throughout the channel. The model supports the idea of stochastic Coulomb blockade [35], where one or more localized states participate in transport through the channel, depending on their positions and the mismatch between their respective quantized energy levels.

\section{TIP INFLUENCE ON THE CONDUCTANCE RESONANCES}

To investigate the formation of the localized states in the channel, we perform SGM measurements, initially at a fixed lateral tip position above the channel [yellow cross in Fig. 3(a)]. We first measure the linear conductance $G$ as a function of tip voltage $V_{\text {tip }}$ and displacement field $D$. The resulting data depicted in Fig. 2(a) show that the conductance resonances depend linearly on both tip voltage and displacement field as expected from a capacitance argument. The resonances labeled in Fig. 1(c) can be identified using the strong correlation of the peak positions in the displacement field $D$ along a vertical cut at $V_{\text {tip }}=-5 \mathrm{~V}$ with the data in Fig. 1(c). The resonances in Fig. 2(a) are labeled accordingly. We thus observe that the experimental signatures in absence of the tip are recovered for a tip voltage $V_{\text {tip }}=-5 \mathrm{~V}$ in presence of the tip. The tunnel coupling between the localized states in the channel and the leads is adjusted by the tip-induced potential. As a consequence, we observe different peak amplitudes in the absence [Fig. 1(c)] and presence [Fig. 2(a)] of the tip.

The negative slope of the resonances in Fig. 2(a), together with the fact that increasing tip voltage will increase the number of electrons in the channel, confirms that increasing the displacement field increases the electron number in the channel. Moving along the orange dotted line in Fig. 1(b) in the positive $D$ direction, the split gate exactly compensates the electron-accumulating tendency of the back gate in dual-gated regions of the device. In the channel, however, the action of the split gate is reduced to stray-field effects, and, as a consequence, electron accumulation by the back gate gains dominance.

The observation that all resonances in Fig. 2(a) have essentially the same slope indicates that the resonances originate either from one localized state or from few spatially closely spaced localized states. The increasing separation of resonances for increasingly positive tip voltage is in agreement with the same observation in Fig. 1(d).

Individual resonances would die out in Fig. 2(a) with increasing $D$ and decreasing $V_{\text {tip }}$, because the increase in $D$ also acts in reducing the tunnel coupling of the states to the leads, while $V_{\text {tip }}$ locally keeps the localized states resonant with the Fermi level.

The influence of the tip at fixed position on the energy of the localized states allows us to measure Coulomb blockade diamonds as a function of tip voltage and DC-source drain bias as depicted in Fig. 2(b). Similar to Fig. 1(d), we observe Coulomb blockade diamonds of different sizes as well as low-energy excited state resonances outside the diamonds of suppressed conductance. The Coulomb diamonds are consistent with a constant lever arm and the increasing zero-bias peak spacing. From these measurements we extract a tip lever arm $\alpha_{\text {tip }}=0.0023$. This rather small lever arm is the result of the screening action of the split gates.

In order to obtain spatial information about the position of the localized states in the channel, we raster scan the SGM tip in the region above the channel. For these measurements, the channel is formed by the displacement field $D=0.3 \mathrm{~V} / \mathrm{nm}$ and the tip voltage is given by $V_{\text {tip }}=-8 \mathrm{~V}$, which is repulsive for electrons. The resulting measurement is shown in Fig. 3(a). We observe concentric rings centered roughly around the location of the localized states.

The ring shape of the resonances can be understood as follows: Due to the extended electrostatic potential of the tip induced in the channel, a change in the distances between the voltage-biased tip and the localized states has a similar 

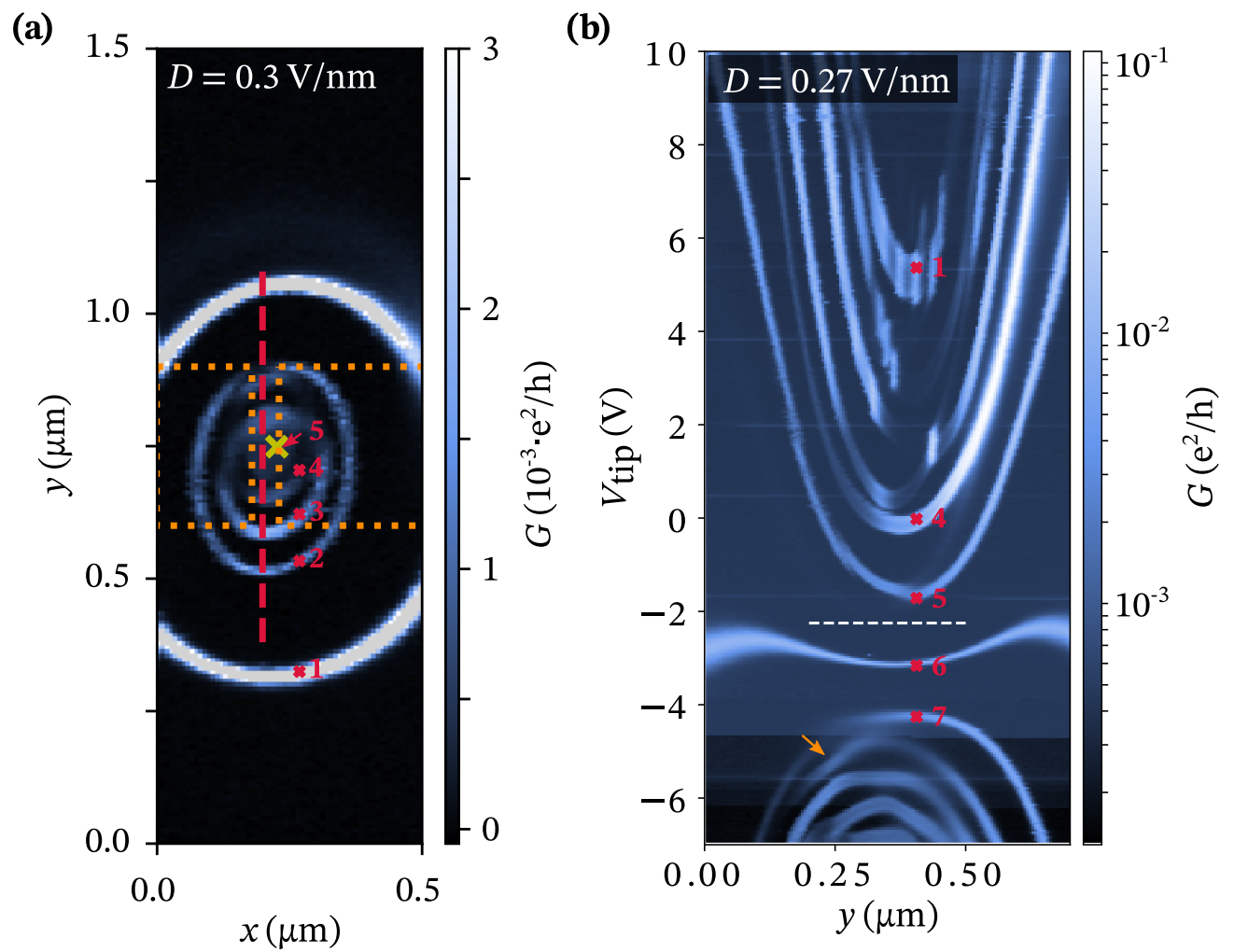

FIG. 3. (a) SGM measurement $\left(V_{\text {tip }}=-8 \mathrm{~V}, D=0.3 \mathrm{~V} / \mathrm{nm}\right)$ above the channel depicting concentric rings around the middle of the channel. The outlines of the split gates are denoted by the orange dashed lines. The yellow cross marks the tip position at which the data in Fig. 2 are obtained. Conductance values exceeding the upper limit of the color scale are depicted in grey. (b) Logarithmic plot of the linear conductance $G\left(y, V_{\text {tip }}\right)$ along the red dashed line in Fig. 2(a) for a displacement field $D=0.27 \mathrm{~V} / \mathrm{nm}$, different from that in (a). The white dashed line denotes the approximate position of the split gates. The orange arrow exemplarily marks the position of an avoided crossing.

effect on the energy levels as a change in the tip voltage. The absolute separation between the tip and the localized states therefore allows us to tune the energy levels of the latter with respect to the Fermi energy, and thereby to observe conductance resonances. The shape of a specific resonant ring is dictated by the geometry of the potential induced by the tip in the graphene plane, which is itself determined by the tip and gate geometries. The spherical geometry of the tip together with the extended long edges of the split gates leads to a nearly elliptic shape of individual resonant rings, with the long axis of the ellipses almost parallel to the channel axis.

For rings at large lateral tip-localized state separations, the position of the localized states cannot be determined with great accuracy. However, bringing the tip closer to the center of the channel, we observe three rings centered at slightly different positions close to the center of the channel (for details, see Appendix A). This observation confirms that resonant transport occurs through states localized in the channel midway along the channel axis.

To ascertain the peak number belonging to these ellipses, we extract the peak occurring at $D \approx 0.3 \mathrm{~V} / \mathrm{nm}$ and $V_{\text {tip }}=$ $-8 \mathrm{~V}$ in Fig. 2(a) as peak number 5. As the measurement depicted in Fig. 2(a) was obtained at the tip position marked by the yellow cross in Fig. 3(a), the innermost ellipse close to this tip position thus corresponds to peak 5 . Due to the decrease of the tip-potential influence for increasing tip-localized state distances, this allows us to identify the remaining ellipses from the inner ellipse outwards as labeled in Fig. 3(a).

To get a better understanding of the influence of the tip potential and the position of the localized states that dominate transport, we measure the conductance $G\left(y, V_{\text {tip }}\right)$ along the channel axis [red dashed line in Fig. 3(a)]. The gating effect of the tip gives rise to the parabolic resonances observed in Fig. 3(b). The curvature of those parabolas changes from convex to concave at the symmetry line $V_{\text {tip }} \approx-3 \mathrm{~V}$, which we call the least-invasive tip voltage following previous experiments on GaAs [36]. We attribute the offset of the latter from zero to the difference in the work functions between the PtIr tip and the sample. The change from convex to concave in the resonance at $V_{\text {tip }}=-3 \mathrm{~V}$ occurs exactly at the outer corners of the channel gates, outlined by the white dashed line. Following each parabola individually, we observe that their extrema occur at different tip positions $y$ along the dashed red line (see Appendix B for details). This is in good agreement with the observation that the concentric rings in Fig. 3(a) are centered at different positions in the center of the channel. Resonances at tip voltages below the least-invasive voltage $V_{\text {tip }}^{\mathrm{LI}}=-3 \mathrm{~V}$ unambiguously show avoided crossings [one of them marked exemplarily by the orange arrow in Fig. 3(b)] indicating mutual interactions between the respective 

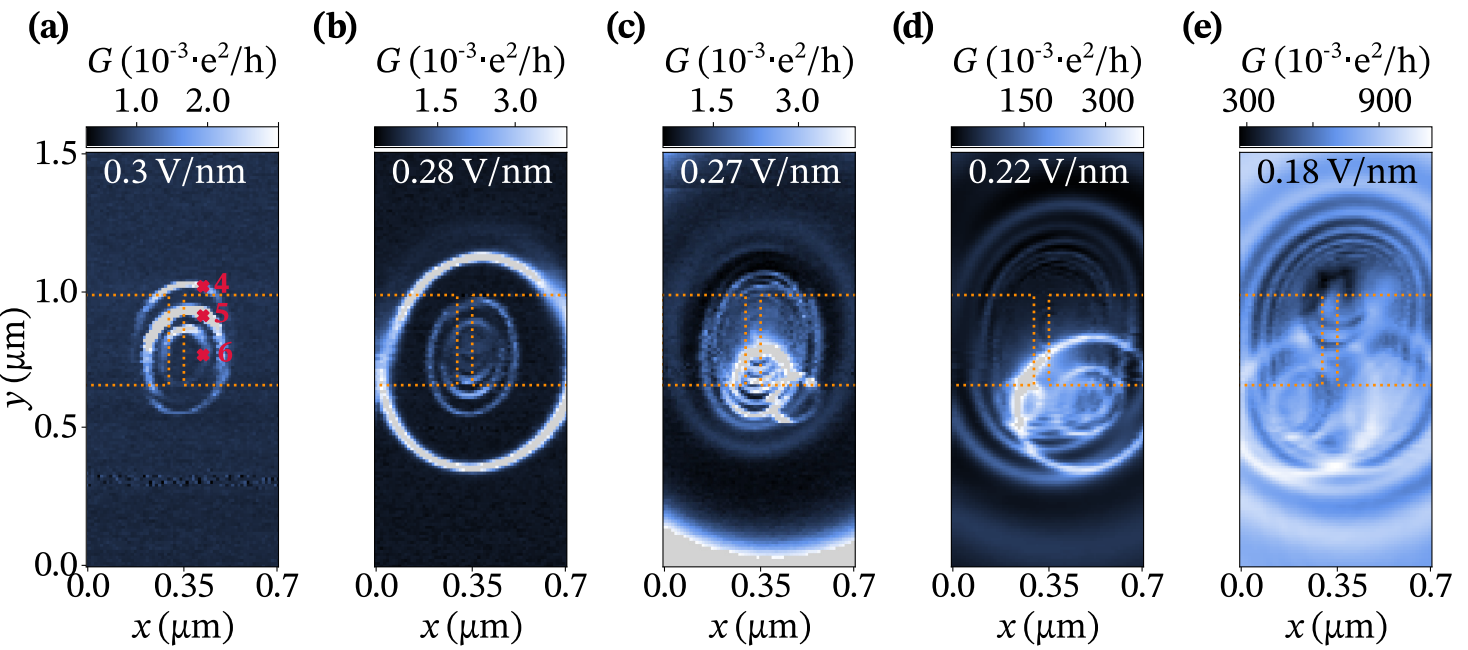

FIG. 4. Evolution of the SGM measurements $\left(V_{\text {tip }}=-9 \mathrm{~V}\right)$ as a function of displacement field $D$ (upper white text, inset). The orange dashed lines denote the outlines of the split gates. Conductance values exceeding the upper limit of the color scale are depicted in grey.

localized states. This observation further strengthens the notion of stochastic Coulomb blockade.

In order to investigate the localized states observed in Fig. 2(a) for specific stepwise decreased displacement fields, we perform two-dimensional scans for $V_{\text {tip }}=-9 \mathrm{~V}$. The results are depicted in Fig. 4. The leftmost figure [Fig. 4(a)] depicts the measurement for $D=0.3 \mathrm{~V} / \mathrm{nm}$, and shows three concentric ellipses centered at approximately the same position close to the middle of the channel. Identifying the peaks with the method used in Fig. 3(a), the inner ellipse corresponds to peak number 6 . This allows us to label the middle and outer rings with peak numbers 5 and 4 , respectively. The observation that ellipses are centered in one single location could correspond to a single localized state in the center of the channel.

Decreasing the displacement field to $D=0.28 \mathrm{~V} / \mathrm{nm}$ [Fig. 4(b)], we observe (at least) two additional elliptical resonances. The smaller ellipses close to the center of the channel are centered at two different positions within the channel, whereas the larger outer ellipses seem to be centered at the same position within the channel. A reliable identification of the peak numbers in this configuration $(D=0.28 \mathrm{~V} / \mathrm{nm}$, $\left.\mathrm{V}_{\text {tip }}=-9 \mathrm{~V}\right)$ is hindered by the very dense peak spacing in this configuration in Fig. 2(a). However, similar to the SGM measurement in Fig. 3(a), the concentric rings are preferentially centered at positions close to the middle of the channel rather than in the outer channel regions.

Decreasing the displacement field even further, more and more concentric ellipses appear in the SGM measurements [Figs. 4(c)-4(e)]. In addition to the ellipses centered in the middle of the channel, we observe rings centered at additional positions close to the edges of the split gates for displacement fields $D \leqslant 0.27 \mathrm{~V} / \mathrm{nm}$ [Figs. 4(c)-4(e)]. The first of those rings appears at $D=0.27 \mathrm{~V} / \mathrm{nm}$ [Fig. 4(c)] and their number increases for even smaller displacement fields $[D=0.22 \mathrm{~V} / \mathrm{nm}$ in Fig. $4(\mathrm{~d})$ and $D=0.18 \mathrm{~V} / \mathrm{nm}$ in Fig. 4(e)]. We interpret their appearance as evidence that the conductance below the split gates starts to increase at these displacement fields. This increase appears to be spatially inhomogeneous, dominated by individual localized states.

\section{CONCLUSION}

We presented scanning gate measurements on a gatedefined channel in high-quality bilayer graphene. The scanning gate images reveal the occurrence of stochastic Coulomb blockade in the conductance through the channel at various displacement fields. We have shown that the conjunction of the split gates forming the channel and the back gate is sufficient to render the $50 \mathrm{~nm}$ wide channel insulating. Our data suggest that the charge neutrality point of the channel in our sample is located at positive displacement fields. This suggests that transport in the region below $D=0.34 \mathrm{~V} / \mathrm{nm}$ occurs through states of a character dominated by the valence band. In the situation of strongly suppressed conductance, localized states are most likely to be observed close to the middle of the channel. If the displacement field becomes too small, the dual-gated regions start to leak. This leakage is again dominated by the local inhomogeneity of the potential.

\section{ACKNOWLEDGMENTS}

We thank Peter Märki as well as the staff of the ETH clean-room facility FIRST for their technical support. We also acknowledge financial support by the Eidgenössische Technische Hochschule (ETH) Zürich, Grant No. ETH-38 17-2, the Swiss National Science Foundation through the National Center of Competence in Research (NCCR) Quantum Science and Technology, and the European Graphene Flagship. Growth of hexagonal boron nitride crystals was supported by the Elemental Strategy Initiative conducted by the Ministry of Education, Culture, Sports, Science and Technology (MEXT), Japan, Grant No. JPMXP0112101001, the Japan Society for the Promotion of Science (JSPS), Grant No. JP20H00354, and the Japan Science and Technology Agency (JST) through Core Research for Evolutional Science and Technology (CREST), Grant No. JPMJCR15F3. 


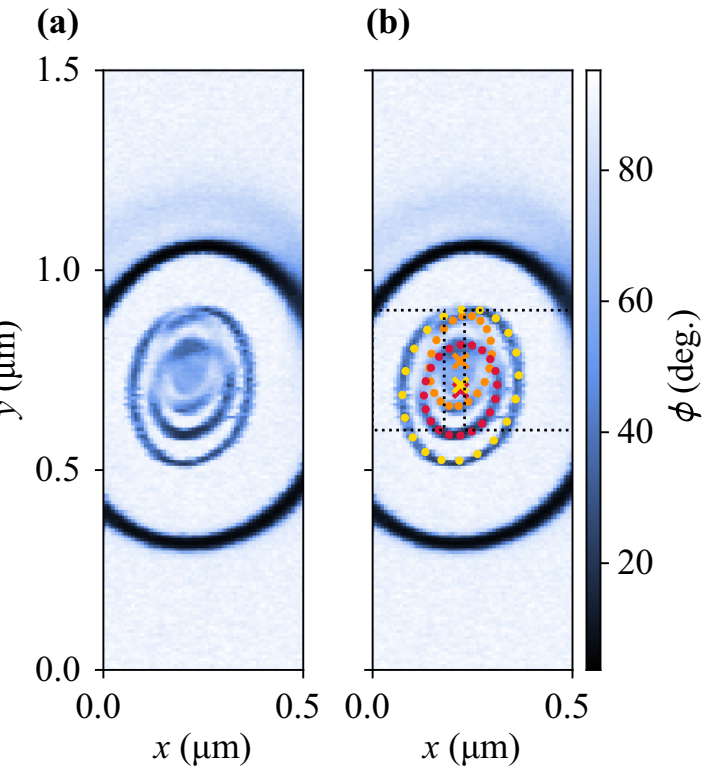

FIG. 5. SGM measurement of the phase of the conductance above the channel. (a) Raw data depicting elliptic features centered at different positions. (b) Same data as in (a) but with guides to the eye. The dotted yellow, red, and orange ellipses denote the smallest ellipses centered at the three different center points marked by crosses of the same color. The outlines of the split gates are denoted by the black dashed lines.

\section{APPENDIX A: DETERMINATION OF THE CENTER OF THE ELLIPSES}

The SGM measurement in Fig. 3(a) depicts elliptic features which are centered around three different locations within the channel. To extract the exact position of these center points, we plot the lock-in measured phase of the conductance signal as a function of the tip position above the channel in Fig. 5. Throughout all measurements, the presence of cable capacities in the measurement setup connected in parallel to the sample impedance gives rise to a fixed capacitive current flowing in parallel to the sample. This capacitive current appears as a quadrature component in our lock-in measurements. The varying strength of the in-phase current through the sample as compared to the constant capacitive current gives rise to the phase shifts observed in Fig. 5. If the channel is completely insulating (implying that no energy level of a localized state is in resonance with the reservoirs), the conductance through the sample is suppressed. In this situation, the capacitive current is important and gives rise to a phase shift of up to $90^{\circ}$ in the phase of the conductance signal. Once an energy level is shifted into resonance with the reservoirs by the tip, the current through the sample increases. This decreases the significance of the capacitive current which results in a reduction of the measured phase shift. Thus Fig. 5 depicts the same elliptic resonances observed in Fig. 3(a). However, due to the nature of the phase signal, small signatures difficult to see in Fig. 3(a) due to variations in the conductance amplitude for various peaks are easier to distinguish. The raw data in Fig. 5(a) depict elliptic features centered at three distinct locations within the channel. To extract the exact po-

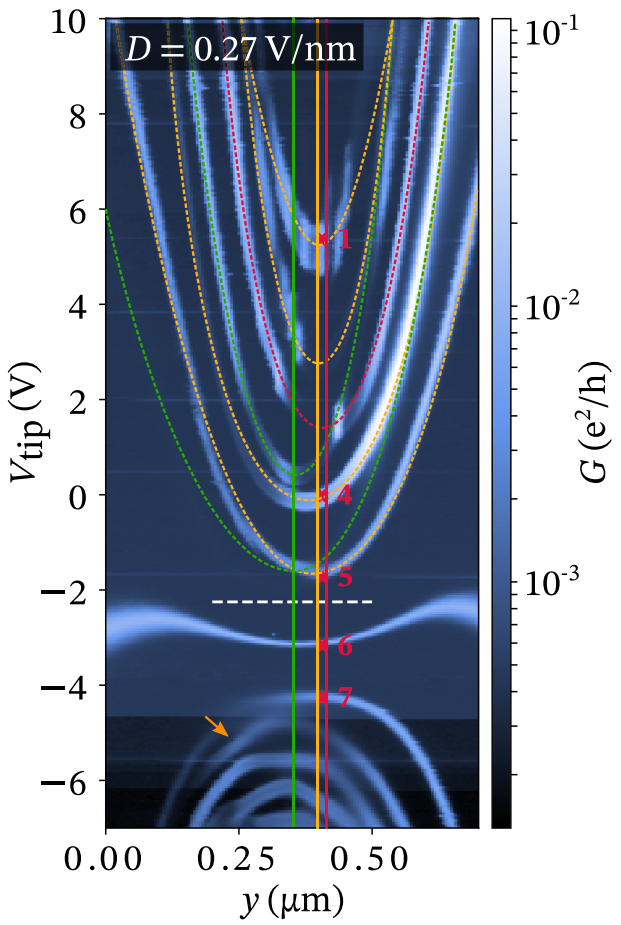

FIG. 6. Logarithmic plot of the linear conductance $G\left(y, V_{\text {tip }}\right)$ in Fig. 3(b) along the red dashed line in Fig. 3(a). The dashed parabolic guides to the eyes follow the observed experimental parabolic features individually. The latter can be separated into groups (denoted by different colors) centering at different positions within the channel (denoted by the vertical lines through the center points of the parabolas belonging to each group).

sition of the centers of these ellipses, we model three ellipses around a center point $\left(x_{c, i}, y_{c, i}\right)$ and rotated by an angle $\theta$ according to

$$
\left(\begin{array}{l}
x_{i} \\
y_{i}
\end{array}\right)=\left(\begin{array}{l}
a_{i} \cos (\theta) \cos (t)-b_{i} \sin (\theta) \sin (t)+x_{c, i} \\
a_{i} \sin (\theta) \cos (t)+b_{i} \cos (\theta) \sin (t)+y_{c, i}
\end{array}\right) .
$$

Here, $i \in(0,1,2)$ denotes the index of the ellipse, $a_{i}$ is half the width of the ellipse, $b_{i}$ is half the height of the ellipse, and $t \in[0,2 \pi]$. Using a rotation angle $\theta=0.25 \mathrm{rad} \approx 14^{\circ}$, this allows us to extract the three center points of the ellipses [yellow, red, and orange dotted elliptic guides to the eyes in Fig. 5(b)] marked by the yellow, red, and orange crosses in Fig. 5(b). These center points are positioned close to the center of the channel between the split gates outlined by the black dashed lines in Fig. 5(b).

\section{APPENDIX B: POSITION OF LOCALIZED STATES FROM FIG. 3(b)}

The parabolas depicted in Fig. 3(b) arise due to the gating effect of the tip voltage and position onto at least three individual localized states located at different positions within the channel. To highlight this observation, we plot the data depicted in Fig. 3(b) with additional parabolic guides to the eyes in Fig. 6. Following each parabolic feature individually [dashed parabolic guides to the eyes in Fig. 6], we can 
distinguish three different center points of the parabolas. Those points are marked by the vertical lines in Fig. 6 and correspond to different localized states positioned at various positions along the channel. Parabolas arising due to the influence of the tip on the same localized state are marked in the same color.
[1] B. Trauzettel, D. V. Bulaev, D. Loss, and G. Burkard, Spin qubits in graphene quantum dots, Nat. Phys. 3, 192 (2007).

[2] L. A. Ponomarenko, F. Schedin, M. I. Katsnelson, R. Yang, E. W. Hill, K. S. Novoselov, and A. K. Geim, Chaotic Dirac billiard in graphene quantum dots, Science 320, 356 (2008).

[3] C. Stampfer, E. Schurtenberger, F. Molitor, J. Güttinger, T. Ihn, and K. Ensslin, Tunable graphene single electron transistor, Nano Lett. 8, 2378 (2008).

[4] D. Bischoff, A. Varlet, P. Simonet, T. Ihn, and K. Ensslin, Electronic triple-dot transport through a bilayer graphene island with ultrasmall constrictions, New J. Phys. 15, 083029 (2013).

[5] D. Bischoff, A. Varlet, P. Simonet, M. Eich, H. C. Overweg, T. Ihn, and K. Ensslin, Localized charge carriers in graphene nanodevices, Appl. Phys. Rev. 2, 031301 (2015).

[6] C. R. Dean, A. F. Young, I. Meric, C. Lee, L. Wang, S. Sorgenfrei, K. Watanabe, T. Taniguchi, P. Kim, K. L. Shepard, and J. Hone, Boron nitride substrates for high-quality graphene electronics, Nat. Nanotech. 5, 722 (2010).

[7] L. Wang, I. Meric, P. Y. Huang, Q. Gao, Y. Gao, H. Tran, T. Taniguchi, K. Watanabe, L. M. Campos, D. A. Muller, J. Guo, P. Kim, J. Hone, K. L. Shepard, and C. R. Dean, One-dimensional electrical contact to a two-dimensional material, Science $\mathbf{3 4 2}$, 614 (2013)

[8] H. Overweg, H. Eggimann, X. Chen, S. Slizovskiy, M. Eich, R. Pisoni, Y. Lee, P. Rickhaus, K. Watanabe, T. Taniguchi, V. Fal'ko, T. Ihn, and K. Ensslin, Electrostatically induced quantum point contacts in bilayer graphene, Nano Lett. 18, 553 (2018).

[9] J. B. Oostinga, H. B. Heersche, X. Liu, A. F. Morpurgo, and L. M. K. Vandersypen, Gate-induced insulating state in bilayer graphene devices, Nat. Mater. 7, 151 (2008).

[10] V. Fal'ko, Quantum information on chicken wire, Nat. Phys. 3, 151 (2007)

[11] J. M. Pereira, P. Vasilopoulos, and F. M. Peeters, Tunable quantum dots in bilayer graphene, Nano Lett. 7, 946 (2007).

[12] L. Banszerus, B. Frohn, T. Fabian, S. Somanchi, A. Epping, M. Müller, D. Neumaier, K. Watanabe, T. Taniguchi, F. Libisch, B. Beschoten, F. Hassler, and C. Stampfer, Observation of the Spin-Orbit Gap in Bilayer Graphene by One-Dimensional Ballistic Transport, Phys. Rev. Lett. 124, 177701 (2020).

[13] M. Eich, F. Herman, R. Pisoni, H. Overweg, A. Kurzmann, Y. Lee, P. Rickhaus, K. Watanabe, T. Taniguchi, M. Sigrist, T. Ihn, and K. Ensslin, Spin and Valley States in Gate-Defined Bilayer Graphene Quantum Dots, Phys. Rev. X 8, 031023 (2018).

[14] L. Banszerus, S. Möller, E. Icking, K. Watanabe, T. Taniguchi, C. Volk, and C. Stampfer, Single-electron double quantum dots in bilayer graphene, Nano Lett. 20, 2005 (2020).

[15] Y. Lee, A. Knothe, H. Overweg, M. Eich, C. Gold, A. Kurzmann, V. Klasovika, T. Taniguchi, K. Wantanabe, V. Fal'ko, T. Ihn, K. Ensslin, and P. Rickhaus, Tunable Valley Splitting due to Topological Orbital Magnetic Moment in Bilayer Graphene Quantum Point Contacts, Phys. Rev. Lett. 124, 126802 (2020).
[16] A. Kurzmann, M. Eich, H. Overweg, M. Mangold, F. Herman, P. Rickhaus, R. Pisoni, Y. Lee, R. Garreis, C. Tong, K. Watanabe, T. Taniguchi, K. Ensslin, and T. Ihn, Excited States in Bilayer Graphene Quantum Dots, Phys. Rev. Lett. 123, 026803 (2019).

[17] A. Kurzmann, H. Overweg, M. Eich, A. Pally, P. Rickhaus, R. Pisoni, Y. Lee, K. Watanabe, T. Taniguchi, T. Ihn, and K. Ensslin, Charge detection in gate-defined bilayer graphene quantum dots, Nano Lett. 19, 5216 (2019).

[18] N. M. Freitag, L. A. Chizhova, P. Nemes-Incze, C. R. Woods, R. V. Gorbachev, Y. Cao, A. K. Geim, K. S. Novoselov, J. Burgdörfer, F. Libisch, and M. Morgenstern, Electrostatically confined monolayer graphene quantum dots with orbital and valley splittings, Nano Lett. 16, 5798 (2016).

[19] S. Jung, G. M. Rutter, N. N. Klimov, D. B. Newell, I. Calizo, A. R. Hight-Walker, N. B. Zhitenev, and J. A. Stroscio, Evolution of microscopic localization in graphene in a magnetic field from scattering resonances to quantum dots, Nat. Phys. 7, 245 (2011).

[20] A. C. Bleszynski, F. A. Zwanenburg, R. M. Westervelt, A. L. Roest, E. P. A. M. Bakkers, and L. P. Kouwenhoven, Scanned probe imaging of quantum dots inside InAs nanowires, Nano Lett. 7, 2559 (2007).

[21] P. Fallahi, A. C. Bleszynski, R. M. Westervelt, J. Huang, J. D. Walls, E. J. Heller, M. Hanson, and A. C. Gossard, Imaging a single-electron quantum dot, Nano Lett. 5, 223 (2005).

[22] A. E. Gildemeister, T. Ihn, M. Sigrist, K. Ensslin, D. C. Driscoll, and A. C. Gossard, Measurement of the tip-induced potential in scanning gate experiments, Phys. Rev. B 75, 195338 (2007).

[23] M. T. Woodside and P. L. McEuen, Scanned probe imaging of single-electron charge states in nanotube quantum dots, Science 296, 1098 (2002).

[24] A. Bachtold, M. S. Fuhrer, S. Plyasunov, M. Forero, E. H. Anderson, A. Zettl, and P. L. McEuen, Scanned Probe Microscopy of Electronic Transport in Carbon Nanotubes, Phys. Rev. Lett. 84, 6082 (2000).

[25] M. Bockrath, W. Liang, D. Bozovic, J. H. Hafner, C. M. Lieber, M. Tinkham, and H. Park, Resonant electron scattering by defects in single-walled carbon nanotubes, Science 291, 283 (2001).

[26] S. J. Tans and C. Dekker, Potential modulations along carbon nanotubes, Nature (London) 404, 834 (2000).

[27] M. R. Connolly, K. L. Chiu, A. Lombardo, A. Fasoli, A. C. Ferrari, D. Anderson, G. A. C. Jones, and C. G. Smith, Tilted potential induced coupling of localized states in a graphene nanoconstriction, Phys. Rev. B 83, 115441 (2011).

[28] A. G. F. Garcia, M. König, D. Goldhaber-Gordon, and K. Todd, Scanning gate microscopy of localized states in wide graphene constrictions, Phys. Rev. B 87, 085446 (2013).

[29] S. Schnez, J. Güttinger, M. Huefner, C. Stampfer, K. Ensslin, and T. Ihn, Imaging localized states in graphene nanostructures, Phys. Rev. B 82, 165445 (2010).

[30] Y. Zhang, T.-T. Tang, C. Girit, Z. Hao, M. C. Martin, A. Zettl, M. F. Crommie, Y. R. Shen, and F. Wang, Direct observation of 
a widely tunable bandgap in bilayer graphene, Nature (London) 459, 820 (2009).

[31] M. Eich, Electrostatically defined quantum dots in bilayer graphene, Doctoral thesis, ETH Zurich, 2019, https://doi.org/ 10.3929/ethz-b-000343512.

[32] E. McCann, Electronic properties of monolayer and bilayer graphene, in Graphene Nanoelectronics: Metrology, Synthesis, Properties and Applications, NanoScience and Technology, edited by H. Raza (Springer, Berlin, 2012), pp. 237-275.

[33] H. Overweg, Electrostatically induced nanostructures in bilayer graphene, Doctoral thesis, ETH Zurich, 2018, https:// doi.org/10.3929/ethz-b-000278503.
[34] P. Rickhaus, J. Wallbank, S. Slizovskiy, R. Pisoni, H. Overweg, Y. Lee, M. Eich, M.-H. Liu, K. Watanabe, T. Taniguchi, T. Ihn, and K. Ensslin, Transport through a network of topological channels in twisted bilayer graphene, Nano Lett. 18, 6725 (2018).

[35] M. Kemerink and L. W. Molenkamp, Stochastic Coulomb blockade in a double quantum dot, Appl. Phys. Lett. 65, 1012 (1994).

[36] A. Pioda, S. Kicin, T. Ihn, M. Sigrist, A. Fuhrer, K. Ensslin, A. Weichselbaum, S. E. Ulloa, M. Reinwald, and W. Wegscheider, Spatially Resolved Manipulation of Single Electrons in Quantum dots Using a Scanned Probe, Phys. Rev. Lett. 93, 216801 (2004). 